\title{
Latent tuberculosis in the general practice context
}

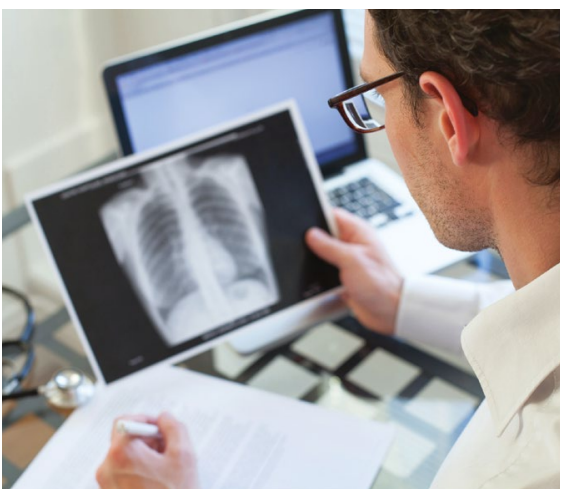

CPD

Justin Denholm, Ann-Marie Baker, Mark Timlin

\section{Background \\ Latent tuberculosis infection (LTBI) is an asymptomatic condition that may progress to active tuberculosis (TB), sometimes decades after exposure. Most people with active TB in Australia have not had recent contact and have been unaware of their risk. Tests for LTBI are available, allowing for diagnosis and preventive therapy to avoid active disease.}

\section{Objective}

The aim of this article is to review current approaches to the diagnosis and management of LTBI, with particular focus on the Australian general practice setting. Groups at elevated risk of having $L T B I$ and progressing to active disease are outlined. Recent research into the prevalence and distribution of LTBI in Australia is reviewed, and Australian guidelines for testing and treatment are summarised.

\section{Discussion}

LTBI occurs in an estimated $5 \%$ of all Australian residents. However, this is a particular issue for those born in TB-endemic countries. Approximately $17 \%$ of all overseas-born Australian residents, but only $0.4 \%$ of Australianborn residents, have LTBI. Appropriate diagnosis and management is an important long-term health promotion activity, and many people with LTBI can be managed safely and effectively in Australian general practice settings.
LATENT TUBERCULOSIS INFECTION (LTBI) occurs after a person has contact with active tuberculosis (TB), when inhaled droplets containing Mycobacterium tuberculosis enter the lungs. ${ }^{1}$ While a proportion of people exposed to TB (especially those with immature or compromised immunity) progress to become unwell soon after exposure, the majority of people spontaneously control the initial infection and enter a state of latency. ${ }^{2}$ LTBI is asymptomatic and may persist lifelong, and people with LTBI remain at increased risk of 'reactivation' and development of active TB in the future.

LTBI is frequently under-recognised within both general and healthcare communities. Community focus groups and healthcare worker surveys conducted by the Victorian Tuberculosis Program have consistently found that general practitioners (GPs) were the preferred source of trusted health information about LTBI, but also that many GPs identified a need for more training and support in providing information confidently. The purpose of this article is to review LTBI, with a particular focus on management in Australian general practice settings.

\section{LTBI prevalence and} distribution in Australia

Although exposure to active TB, and acquisition of LTBI, is uncommon in Australia, approximately one-quarter of the world's population is affected. ${ }^{3}$ Recent work into the prevalence and distribution of LTBI in Australia, based on census data and international studies of TB risk, has highlighted important trends. LTBI affects approximately $5 \%$ of all Australian residents; however, it more commonly affects those born in TB-endemic countries. ${ }^{4}$ Approximately $17 \%$ of all overseas-born Australian residents, but only $0.4 \%$ of Australianborn residents, have LTBI. ${ }^{4}$ Residents in urban areas are also more likely to have LTBI. ${ }^{4}$ Following migration to Australia, people with LTBI have the highest risk of developing active disease in the first five years (approximately 400/100,000/year), but risk persists lifelong at higher rates than for the Australian-born population and may increase with ageing. ${ }^{5,6}$

Although the risk of active disease persists long term, most individuals with LTBI (up to $90 \%$ overall) will never reactivate. ${ }^{5}$ Factors known to increase the risk of active disease include immunosuppressive medications or conditions, such as human immunodeficiency virus; use of tumour necrosis factor- $\alpha$ inhibitors or corticosteroids; diabetes; chemotherapeutic agents and haemodialysis. However, most people who develop active TB in Australia have no specific risk factors for reactivation, and the mechanisms for triggering active disease remain unclear in many cases. 


\section{Diagnosis of LTBI}

Making a diagnosis of LTBI is valuable, because it allows appropriate management to reduce the risk of developing active disease. Two diagnostic tests for LTBI are available in Australia: the tuberculin skin test (TST; also known as the Mantoux test) and the interferon-gamma release assay (IGRA). These tests have a number of similarities and some key differences (Table 1). Both tests assess the cellular immunological response of individuals with a history of possible TB exposure and tend to remain positive indefinitely after infection or disease. In practice, local test accessibility and a history of Bacillus Calmette-Guérin (BCG) vaccination are typically key factors in determining which test should be used, as both are recommended by Australian guidelines. ${ }^{7}$ Importantly, neither test distinguishes between LTBI and active TB disease, so clinical features and other investigations are required to exclude active TB prior to establishing a diagnosis of LTBI. This assessment should include at least a clinical review for any symptoms of active TB (including fever, cough, weight loss or lymphadenopathy, particularly when present for $>3$ weeks) and a chest X-ray.

Australian national guidelines recommend that either a TST or an IGRA may be appropriate for the diagnosis of LTBI. ${ }^{7}$ TSTs are generally preferred for children under the age of five to avoid venepuncture, while IGRAs are typically preferred for those with a history of BCG vaccination. Some patients will be eligible for a Medicare Benefits Schedule (MBS) rebate on IGRAs, including individuals at higher risk of TB exposure (eg those with a history of TB contact) and those at higher risk of developing active TB in the future (eg those with immunosuppressive conditions or treatment, silicosis or undergoing haemodialysis). For individuals not eligible for an MBS rebate, IGRAs typically cost $\$ 60-80$ but may vary between providers.

Some patients may present requesting a test for LTBI, particularly as part of pre-employment or education placement testing. However, as LTBI is an asymptomatic condition, most affected individuals will not spontaneously identify the need for testing. It is therefore important that GPs are alert to patients at risk of past infection, especially those born in countries with high rates of $\mathrm{TB}$, and consider opportunistic testing for people presenting for other reasons. Testing is most valuable soon after migration but may still provide a useful opportunity for TB prevention years after arrival. Of note, the current Australasian Society of Infectious Diseases guidelines recommend screening of all people from a refugee background, preferably within one month of arrival. ${ }^{8}$

\section{Treatment of LTBI}

Several medication regimens are used internationally for the treatment of LTBI, including those based on rifampicin or rifapentine. ${ }^{9}$ In Australia, currently only isoniazid is listed on the Pharmaceutical Benefits Scheme for LTBI treatment, and it is normally prescribed as a once-daily therapy (10 mg/kg, up to $300 \mathrm{mg}$ ) for a duration of 6-9 months. ${ }^{7}$ Isoniazid is generally well tolerated, with Australian treatment series showing high levels of completion and low rates of serious adverse effects. ${ }^{10,11}$ The most common side effects include gastrointestinal upset and acne; hepatotoxicity may also occur during treatment. The risk of hepatotoxicity is higher in older people (particularly those aged $>50$ years) and those with abnormal liver function tests prior to therapy. Peripheral neuropathy has also been described, particularly in those with underlying nutritional deficiencies, and co-prescription of vitamin B6 (pyridoxine) is generally offered. Patients aged $<35$ years with normal liver function tests can be prioritised for treatment in general practice settings, while others at higher risk for adverse effects during treatment can be referred for assessment and management by appropriate local infectious diseases physicians or chest clinics.

\section{Managing LTBI in the general practice setting}

Historically, most management of LTBI has been provided in tertiary care settings, particularly chest clinics and infectious diseases outpatient services. However, such settings may be difficult to access or result in delays in initiating therapy. Reflecting an increasing recognition of the extent of LTBI in the Australian community, a range of approaches to support GPs in safe and effective management exist. The Victorian Tuberculosis Program has produced a series of video tutorials focusing on different aspects of LTBI diagnosis and management in the Australian general practice context. These are available online (refer to Resources). A flowchart highlighting key steps in community-based LTBI management is presented in Figure 1.

After initiating therapy, strong adherence is important for successful outcomes. Ongoing clinical review is needed to help support patients to successfully complete treatment and monitor for any adverse effects of medication. ${ }^{12}$ Support for patients may include provision of educational materials; review from practice nurses and GPs to discuss adherence to

\section{Table 1. Interferon-gamma release assay versus tuberculin skin test}

\begin{tabular}{ll}
\hline Interferon-gamma release assay & Tuberculin skin test \\
\hline - An in vitro laboratory test & - An in vivo clinical test \\
- Measures the patient's white blood & - Measures inflammation (dermal induration) \\
cell cytokine release in response to & in response to injection of purified protein \\
tuberculosis antigens & derivative \\
- A blood test & A subdermal injection that is read \\
- Non-reactive to Bacillus Calmette-Guérin & 72 hours later \\
vaccine & Reactive to Bacillus Calmette-Guérin \\
- Does not decline with treatment & vaccine \\
& Does not decline with treatment \\
\hline
\end{tabular}


treatment, side effects or interruptions; and encouragement of continuation and successful treatment. An important patient consideration is the level of health literacy and proficiency in English, while an important practice consideration is the ability to allocate sufficient appointment time. Assuming these criteria can be met, applying MBS billing item numbers such as a Chronic Disease Management Plan and/ or a Team Care Arrangement can facilitate the required staff resources.

Discussion with, or referral to, a local TB service may be required for some patients at various stages on the LTBI management pathway. In particular, patients with signs or symptoms of active disease, those who are pregnant or those with significant adverse effects from LTBI treatment should be referred for specialist involvement. A good working relationship with an appropriately experienced specialist or clinic is helpful for facilitating timely review, and remote support via telephone or video consultation may supplement safe and convenient care. ${ }^{13}$

Cases 1 and 2 illustrate common scenarios that may be encountered in general practice settings. Although both individuals migrated from countries with a high incidence of TB and have a positive test for LTBI, differences in their comorbidities and ages affect the risk of reactivation and adverse effects with treatment. Consideration of these factors helps to determine whether non-GP specialist review is appropriate prior to initiation of LTBI therapy.

\section{Practice tips}

Patients are often uncertain about the significance of an LTBI diagnosis and may ask whether there is a risk of transmission to others around them. It is important to reassure patients that LTBI is not contagious, and there is no risk of transmission to others unless reactivation occurs. Patient education should include recognition of the signs and symptoms of reactivation to active $\mathrm{TB}$ to facilitate early treatment in the unlikely event this occurs.

To cater for people who are culturally and linguistically diverse, special attention should be given to medication safety, cultural competence and the ability to work with trained interpreters. The use of interpreters is important

Consider tuberculosis

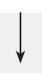

Test for latent tuberculosis infection

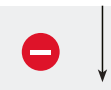

No further action

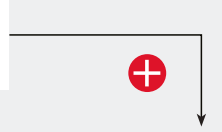

Exclude active disease (symptoms and chest X-ray)

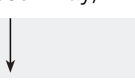

Aged $<35$ years and normal liver function test

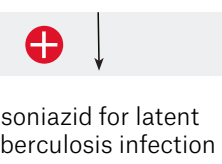
tuberculosis infection

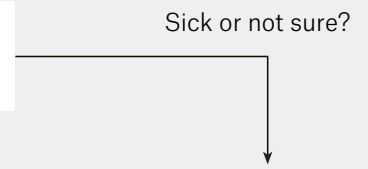
advice

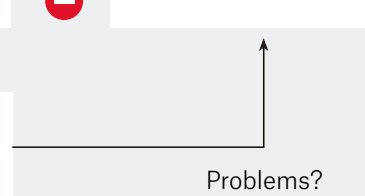

Figure 1. Flowchart for general practice management of suspected latent tuberculosis infection

to ensure appropriate communication and understanding, and free telephone interpreting services are available through the Translating and Interpreting Service (TIS National). A variety of free online resources are available and may be helpful (refer to Resources).

Each state and territory in Australia has a dedicated TB program, with particular responsibility for management of the clinical and public health issues related to $\mathrm{TB}$. A diagnosis of active TB requires notification to the appropriate jurisdictional service, which will then also arrange for any LTBI testing needed for community members with possible contact.

Patients who present and report being exposed to recent local cases of TB should be discussed directly with relevant jurisdictional TB programs, which are responsible for testing and follow-up in cases of confirmed disease.

\section{CASE 1}

$\mathrm{S}$, aged 22 years, is a male asylum seeker who arrived from Myanmar three months ago. He completes the recommended refugee screening tests, which include an IGRA that is subsequently found to be positive. He is well, with no symptoms suggesting active TB. He remembers that his aunt had TB when he was a child, but he says that he has never been tested for LTBI before. His GP orders a chest $\mathrm{X}$-ray and liver function tests, which are both unremarkable. The GP therefore discusses with $S$ the diagnosis of LTBI and suggests that he consider treatment with daily isoniazid for six months to prevent becoming unwell in future. $S$ agrees and completes a successful course of therapy while continuing his nursing studies.

\section{CASE 2}

$\mathrm{W}$, aged 65 years, is a woman from India who has recently been diagnosed with rheumatoid arthritis. As part of an assessment during consideration of immunosuppressive therapy, she is found to have a positive IGRA. She has a normal chest X-ray and no symptoms of active TB, but is noted to have a history of fatty liver disease; her most 
recent alanine transaminase result is 73 U/L. Her GP discusses the diagnosis of LTBI and the possibility of treatment with $\mathrm{W}$, but recognises her higher risk of adverse effects. The GP therefore arranges for her to be reviewed by a local infectious diseases outpatient clinic, which ultimately monitors her through a course of rifampicin.

\section{Summary}

LTBI is a common condition in Australia but is often unrecognised. As trusted care providers, GPs can play an important part in the diagnosis and management of LTBI in community-based settings. Appropriate and timely management of LTBI is a valuable part of long-term health promotion. This management provides a key contribution to lowering individual risk of TB and reducing Australian TB incidence towards elimination.

\section{Resources}

Many online resources can assist the general practice management of latent tuberculosis. Some examples the authors have found helpful include:

- Video tutorials produced by the Victorian Tuberculosis Program, www.youtube. com/watch?v=3ru7Aw3byRk

- NSW Refugee Health Service Appointment reminder translation tool, www.swslhd.health.nsw.gov.au/ refugee/appointment/

- Easidose-Pictorial prescribing, http://easidose.com

- Service for the Treatment and Rehabilitation of Torture and Trauma Survivors (STARTTS) - Cultural competence in working with people from refugee backgrounds, www.startts. org.au/media/Participant-Booklet_ STARTTS-Cultural-CompetenceWorkshop_Feb2019.pdf

- Tips on working with interpreters, www.tisnational.gov.au/en/

\section{Authors}

Justin Denholm BMed, MBioethics, MPHTM, PhD, FRACP, Medical Director, Victorian Tuberculosis Program, Vic. justin.denholm@mh.org.au
Ann-Marie Baker RN, Grad Dip Community Health, Clinical Nurse Consultant, Victorian Tuberculosis Program, Vic

Mark Timlin MBBS, MPH, MBE, General Practitioner, Member Refugee Health Special Interest Group, Vic Competing interests: None.

Funding: None.

Provenance and peer review: Commissioned, externally peer reviewed.

\section{Acknowledgments}

We are grateful for support from the Department of Health and Human Services (Victoria), the SouthEastern Melbourne Primary Health Network and the National Health and Medical Research Council for development of materials relevant to community management of LTBI.

\section{References}

1. Getahun $H$, Matteelli $A$, Chaisson RE, Raviglione M. Latent Mycobacterium tuberculosis infection. N Eng J Med 2015;372(22):2127-35. doi: 10.1056/NEJMra1405427.

2. Trauer JM, Moyo N, Tay EL, et al. Risk of active tuberculosis in the five years following infection ... 15\%? Chest 2016;149(2):516-25. doi: 10.1016/j. chest.2015.11.017.

3. Houben RM, Dodd PJ. The global burden of latent tuberculosis infection: A re-estimation using mathematical modelling. PLoS Med 2016;13(10):e1002152. doi: 10.1371/journal. pmed.1002152.

4. Dale KD, Trauer JM, Dodd PJ, Houben RMGJ, Denholm JT. Estimating the prevalence of latent tuberculosis in a low-incidence setting: Australia. Eur Respir J 2018;52(6):pii.1801218. doi: 10.1183/13993003.01218-2018.

5. McBryde ES, Denholm JT. Risk of active tuberculosis in immigrants: Effects of age, region of origin and time since arrival in a lowexposure setting. Med J Aust 2012;197(8):458-61. doi: 10.5694/mja12.10035

6. Dale KD, Trauer JM, Dodd PJ, Houben RMGJ, Denholm JT. Estimating long-term tuberculosis reactivation rates in Australian migrants. Clin Infect Dis 2019:pii:ciz569. doi: 10.1093/cid/ciz569.

7. Stock D. National position statement for the management of latent tuberculosis infection Commun Dis Intell Q Rep 2017;41(3):E204-E8

8. Chaves NJ, Paxton G, Biggs BA, et al. Recommendations for comprehensive post-arrival health assessment for people from refugee-like backgrounds. Surry Hills, NSW: Australasian Society for Infectious Diseases, 2016.

9. Fox GJ, Dobler CC, Marais BJ, Denholm JT. Preventive therapy for latent tuberculosis infection - The promise and the challenges. Int J Infect Dis 2017;56:68-76. doi: 10.1016/j. ijid.2016.11.006.

10. Denholm JT, McBryde ES, Eisen D, et al. SIRCLE: A randomised controlled cost comparison of self-administered short-course isoniazid and rifapentine for cost-effective latent tuberculosis eradication. Intern Med J 2017;47(12):1433-36. doi: 10.1111/imj.13601.

11. Denholm JT, McBryde ES, Eisen DP, Penington JS, Chen C, Street AC. Adverse effects of isoniazid preventative therapy for latent tuberculosis infection: A prospective cohort study. Drug Healthc Patient Saf 2014;6:145-49. doi: 10.2147/ DHPS.S68837.

12. Rubinowicz A, Bartlett G, MacGibbon B, et al. Evaluating the role of primary care physicians in the treatment of latent tuberculosis: A population study. Int J Tuberc Lung Dis 2014;18(12):1449-54. doi: 10.5588/ijtld.14.0166.

13. Schulz TR, Richards M, Gasko H, Lohrey Hibbert ME, Biggs BA. Telehealth: Experience of the first 120 consultations delivered from a new refugee telehealth clinic. Int Med J 2014;44(10):981-85. doi: 10.1111/imj.12537. 